\title{
Further development of a method to calculate frequencies of loss of control including their uncertainty
}

\author{
Ben J.M. Ale, Coen van Gulijk, Daniela Hanea, Patrick Hudson, Pei-Hui Lin, \\ Simone Sillem \& Marcel Steenhoek \\ Safety Science, Technical University Delft, Delft, The Netherlands \\ Dan Ababei \\ LightTwist Software, The Netherlands
}

\begin{abstract}
An integrated model for risk in a real-time environment for the hydrocarbon industry based on the CATS model for commercial aviation safety has been further developed. The approach described in earlier papers required Bayesian Belief Nets (BBN) to be developed for each process unit separately. A much more efficient method for developing the continuous BBNs is based on standard building blocks consisting of nodes for a piece of equipment and nodes for potential safety features. Elements of the BBN can be switched on or off as required. From the equipment inventory of a chemical plant a complete BBN can be built automatically. Humans are represented by multiple instances of the same BBN structure, but with different parameters. The resulting computer program calculates the frequency of Loss of Containment Accidents for an existing or a proposed plant including the effects of human behaviour and intervention, including rigorous handling of the uncertainties.
\end{abstract}

\section{INTRODUCTION}

The blow-out and subsequent environmental disaster in the Gulf of Mexico in 2010 have highlighted a number of serious problems in scientific thinking about safety. One of these is that our current thinking about how accidents happen and all the management systems based on that approach, does not appear to enable us to achieve the goal of zero accidents. These problems are particularly clear in the case of what can be described as lowprobability high-consequence accidents which, while quite rare, do not really appear to be reducing in frequency unlike simpler and higher frequency personal accidents (Christou 2008), while their consequences appear to increase. The suggestion is that linear and deterministic models of accident causation are insufficient to catch the residual factors and their interactions (Hudson 2010). This is further complicated by the fact that the safety culture can either augment or diminish the effects of the best of HSE management systems. A deeper and wider-reaching analysis of the risks being taken and run needs to integrate the cultural and regulatory factors into the more accessible technical aspects of risk analysis and assessment.

In 2011 a current project was undertaken to address these issues and thereby to support the vague notion of "Chronic Unease" current in Shell with more concrete information, as this unease is the logical consequence of the combination of "Goal Zero" and the non-zero probability of an accident which has to be accepted given the technical boundary conditions and economic constraints. The approach used in this project is especially suited to the low probability, high consequence events that form the ground for this chronic unease (Beddington 2011).

The development of a Proof Of Concept (POC) was completed in 2012. (Ale et al 2011, Ale et al 2012a, b). It showed that fault-trees and bowties can be integrated with human performance models into a single Bayesian Belief Net (BBN) structure.

In this project several key components of the a particular plant of Shell were modelled by a BBN. These were then integrated with human performance and management models to produce output similar to the safety indicators currently in use by Shell.

Although the project was a success, the method proved to be extremely time consuming. If for the full model BBN structures would have been developed for each vessel, pipe or piece of equipment on the basis of dedicated Layer Of Protection Analyses (LOPA), Hazard and 
Operability studies (HAZOP), and/or similar studies, the necessary effort would be prohibitive for the intended use of the model: a living risk model of a chemical plant or site as whole. Thus the approach was reconsidered. It was decided to produce a more generic type of modelling based on a limited set of archetypical items in a chemical plant such that these could be modelled based on existing "Bow-Tie" exercises in Shell. The advantage of this approach would be that in concrete circumstances the model could be easily adapted. In the definition of these archetypes we stay close to the definitions of standard failures in the Purple Book (Ale \& Uijtdehaag 1999). The Purple Book in its turn is based on the approach taken in the more popular risk assessment methods and computer packages.

\section{MODEL STRUCTURE}

The main structure of the model keeps the four layers that have been developed in the Causal Model for Air Transport Safety-CATS - (Ale et al 2009a, b) and in the POC model. These layers are:

1. Management layer. In this layer all the management activities that influence human behavior in the company are positioned.

2. Human layer. In this layer all human actions are positioned. These actions influence the technical behavior of the plant. It should be noted that management consist of people as well. This means that the human model may contain "managers" which connect to layer nr 1.

3. Hardware layer. In this layer the technical performance of a plan, installation or any equipment is positioned. This performance is influenced by the performance of humans such as operators and maintenance technicians who may be employed by Shell directly of by contractors hired by Shell. The hardware layer is the last layer before - or below - the centre or "top" event. For the current development the top event is a Loss Of Containment (LOC).

4. Consequence layer. In this layer the consequences of a LOC are positioned. These could be physical effects or damages but also KPI's or any other ultimate measure of performance other than the probability of a LOC.

Accident sequences are modelled along the lines of the Bow-Tie, with potentially multiple causes leading to a defined centre event, which in turn can have multiple consequences. However contrary to the classical Bow-Tie approach a BBN allows interdependencies between events and barrier failures to be modelled directly.

\subsection{The bow-tie centre}

Since all events in a BBN have distributed properties the centre event has no single "point" measure. The centre event is defined as the probability distribution of the frequency of release. The consequence of such a release also depends on many other parameters such as duration (which implies a rate, given the total quantity), the physical state of the material released, the location, the meteorological conditions and the nature of the material. These are the standard inputs of any consequence model. These consequence models are components of risk quantification models such as SAFETI (Ale \& Whitehouse 1984). The implementation of these in distributed, probabilistic form is deferred to a later stage of development. In this project we concentrate on finding the probability distribution of the frequency of a LOC.

A company such as Shell has many sites. Each site may have many activities such as production, storage, loading and unloading. To decompose such a large system in building blocks that can have multiple uses the method employed is described in the next sections. The design is meant to be recursive. This means that larger units or plants can be represented by the same structure of smaller parts of a plant. Also the building blocks of safeguards or barriers have been "standardized", to allow multiple use of the same building blocks.

\subsection{Site and company}

The highest level of aggregation in the model is the site or in the case a company has multiple sites the company as a whole. The ultimate goal is to be able to build a risk profile for a company in the form of an FN curve or Risk Matrix and derived indicators such as the expected Fatal Accident Rate (FAR) and Loss Time Injury rate (LTI). It should be borne in mind that the FAR and the LTI are distributed quantities also. If the variability of these quantities can be calculated the meaning of a high or a low value can be better interpreted. The value of a FAR, a LTI or the frequency of loss of containment incidents as an indicator for improving or deteriorating risk and risk management depends for instance on the relationship between the mean time between small incidents and the estimated mean time between catastrophes and on the variability thereof or the uncertainty in the estimates.

\subsection{The plant}

The basic unit of analysis is called a PLANT. A plant can be a distillation train that makes crude oil into separate chemicals and fuels. It can be a chemical reactor with the associated preparation 
and post processing equipement, it can be the loading and unloading facility. The demarcation between plants on a site is a matter of choice and convenience. Although on many modern chemical sites all plants influence each other, plants can still be distinguished, for instance by the fact that they can be isolated form the other plant on the site, can be shut down while the rest keeps operating or by the fact that the transfer of material is restricted to a single component, such as ethylene or styrene. A good division can be made if the plant can be operated in isolation. Each plant is considered to be composed of units.

\subsection{The unit}

A unit consists of coherent pieces of equipment that are meant to perform a single operation. Examples are a set of storage vessels where one vessel is kept as the emergency dump vessel for the others; a distillation column with its heaters and condensers; a reactor with pumps, heating or cooling; a loading dock with loading arms for road tankers. Each unit consists of vessel, pipes and miscellaneous components.

\subsection{The vessel}

A vessel is a single volume designed to hold material. This material can be a single chemical but also a mixture such as crude, or hydrocarbons with a range of boiling points or a mixture of chemicals that are meant to react. The vessel is the focal point for the process parameter definitions and the definition of the safe operating envelope of these parameters. These parameters are:

\section{P (Pressure) \\ $\mathrm{T}$ (Temperature) \\ C (Chemical composition) \\ I (Inventory)}

For the purpose of subsequent consequence analysis it has proven to be handy when a number of standard types of vessels are defined (Ale \& Whitehouse, 1984):

1. Atmospheric: The material is under atmospheric pressure and temperature. This type of vessel is not designed to withstand a pressure above atmospheric or excessive temperatures.

2. Elevated temperature. The pressure in these vessels is designed to be atmospheric but the chemicals in it are heated (e.g. to maintain fluidness).

3. Pressure, gas only. The pressure is elevated but no liquids are present. Typically these are vessels containing Hydrogen or materials with similar low critical temperatures.
4. Two Phase. These vessels contain materials in equilibrium with their vapour pressure, which is higher than atmospheric. Typically these are vessels containing LPG's but also reactors at high pressure and temperatures.

5. Refrigerated. The materials in these vessels are kept liquid by refrigeration. They have to be designed for low temperatures and usually cannot withstand any pressure significantly above atmospheric.

The initial estimate of the failure frequency of these vessels depend on this type (Ale \& Uijtdehaag, 1999).

\subsection{Components}

Components are "small" items that support the function of the vessels. They are incorporated because they are part of the containment system and their existence can contribute to the probability of a LOC. The current list of components considered is:
1. Pumps
2. Flanges
3. Welds
4. Bends
5. Bellows.

\subsection{Valves}

Valves are a special component because they can fail in their function but can also fail as part of the containment system. There for Valves come in three types:

1. Shut off

2. Excess flow

3. Non-return.

\subsection{Pipes}

Pipes connect the vessels inside a unit, but also the units in a plant and the plants on a site. Their specifications include:
$\mathrm{T}$ (Temperature)
P (Pressure)
L (Length)
D (Diameter)
C (Composition)
NF (Normal Flow rate).

\subsection{Inventory}

Shell maintains an equipment data-base which holds the specifications of all material items in any plant. The database has a structure that 
differs from the structure need in the final analysis package. They distinguish:

$\begin{array}{ll}\text { 1. Vessels HC only } & \text { V-HC } \\ \text { 2. Vessels L-L separator } & \text { V-LL } \\ \text { 3. Reactors } & \text { R-GEN } \\ \text { 4. Pipework } & \text { PW } \\ \text { 5. Centrifugal pump } & \text { P-C } \\ \text { 6. Reciprocating pump } & \text { P-R } \\ \text { 7. Furnace } & \text { F-GEN } \\ \text { 8. Fin-fan coolers } & \text { E-FF } \\ \text { 9. Tubular heat exchanger } & \text { E-TS } \\ \text { 10. Centrifugal compressor } & \text { C-C } \\ \text { 11. Reciprocating compressor } & \text { C-R }\end{array}$

The information includes the type and location of any valves and the type, location and function of any safeguard installed. It can easily be seen that once the distribution of the probability of failure is defined the two subdivisions can be connected. In a later stage when the associated distribution of the source strength is defined a connection can be made with the consequence analysis programs.

\section{SPECIFICATIONS}

When a risk analysis is performed aimed at calculating numbers of people affected and their location, precise definitions of locations of all elements of a site is necessary. At this stage of development however the mere potential of loss of containment is a sufficient result. However the length of pipes influences the probability of failure and so does the diameter.

The extent to which changes in the ultimate safety state of the company, or the plant can be traced back to changes in plant parameters, in human behavior and in management actions depend on the granularity of the modeling chose. In each instance it can be chosen to model say a group of vessels or all vessels in a plant as a population of vessels with distributions for the relevant properties. In that case this population would constitute a single node in the BBN. Or it can be chosen to model one or more or even each vessel separately, in which case multiple instances of the vessel $\mathrm{BBN}$ structure would be put in the BBN, each with the parameters specific for the vessel. Typically one would choose to model reactors and certain separation equipment separately and the rest of the vessels as a population.

It is obvious that the granularity of the results of a risk evaluation and the precision and usability these depend on the granularity of the specifications of the site. If one were to desire to know the effect on a site's Key Performance Indicators (KPI)s of a particular management decision on the maintenance of one particular reactor, one needs to model that reactor explicitly. In many cases however it is sufficient to know the nature of the population of certain items such as the range of and distribution over size of vessels.

There for in this section it is described how the specifications of equipment should be compiled to arrive at a minimal granularity of results thereby maintaining the accuracy of the average probability of LOCs as calculated on the basis of the specifications in the Purple Book.

\subsection{Safeguards}

Every parameter such as temperature of pressure of any piece of equipment, the excursion of which could present a safety problem, i.e. lead to a LOC can be protected by a safety system or barrier. Safeguards are the mechanisms that keep these barriers in working order. As the top event is loss of containment in this study only barriers are considered in the causal pathway towards this type of event. Barriers that have the purpose of protecting the quality of products or the rate of production are not considered. The minimum composition of a safeguard is a detector, an alarm, some logic that translates the signal from the detector into the activation of an actuator and the actuator that supplies the barrier.

The diagram depicting a BBN in Figure 1 is typical for such a SIL (Safety Integrity Level) 1 protection: a detector, an alarm, human or computer logic and intervention and an action to remedy the situation combine with the initiating event to lead to the probability of failure of the barrier. The initiating event is a parameter excursion.

Such a system can be simple such as a pressure relief valve in which the detector is the spring, the logic is the physics of the spring and the actuator is the spring as well. The system can also be complicated. The detector can set off an audible alarm. The logic runs through this alarm, a human being who has to hear the alarm, interpret it, design an action and activate some equipment. In all these cases the alarm system is in principle the same.

Within Shell in a two stage or SIL 2 barrier system, the logic of the first barrier is usually provided by a human, the operator, while the second stage is usually automated. So the usual sequence (with something that can go too high as example) is: parameter high $\rightarrow$ High Alarm $->$ operator intervention $\rightarrow$ High High Alarm (if intervention fails) -> automated trip. This structure can also be thought of as consisting of two basic alarm structure as in Figure 2, which would be the generic SIL 2 barrier system typical for Shell.

With these barrier systems defined the plant items can be specified. 


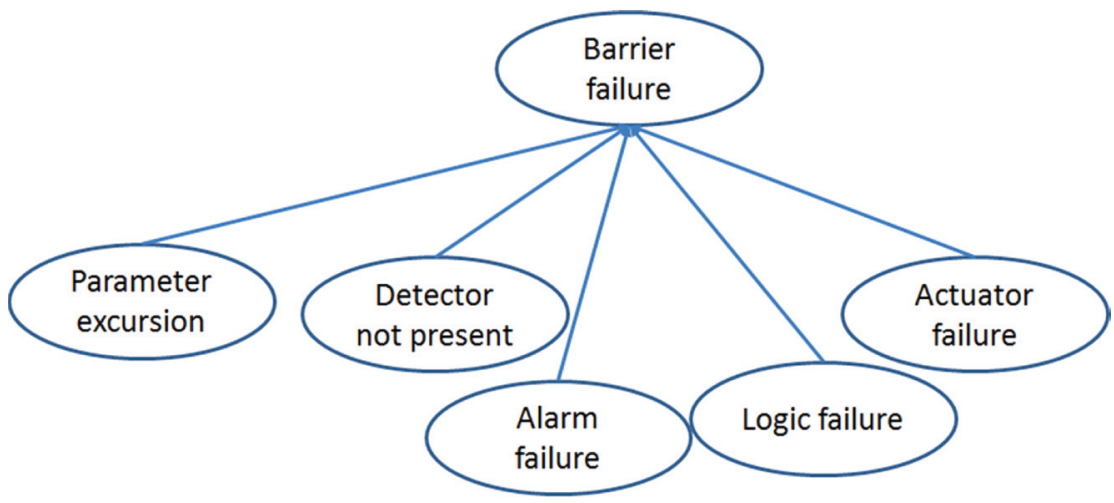

Figure 1. One layer protection system.

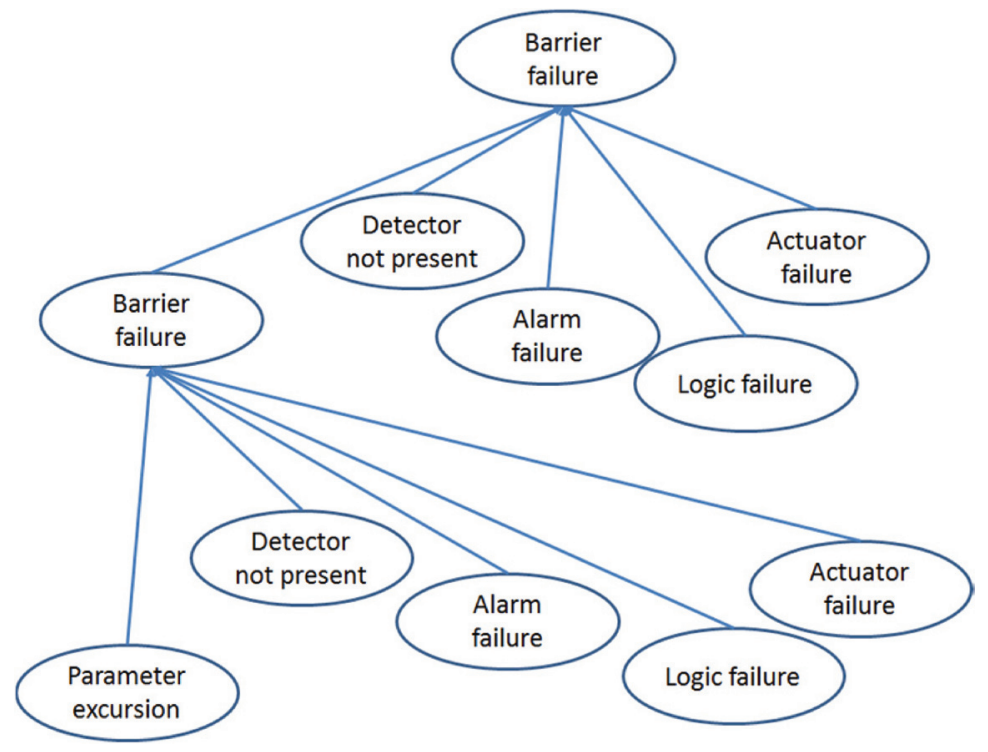

Figure 2. BBN for a typical SIL 2 barrier system.

\subsection{Vessel and pipe level}

The highest granularity in the final result, which also needs the most effort, is the result of specification at the vessel level. Even if this level of granularity is not required, it is necessary to define each type of vessel in the list at least one generic lay-out consisting of a vessel, control mechanisms for pressure and/or temperature and for content or level. In addition devices for filling and end emptying need to be specified in general. The general devices do not necessarily have to be present for all vessels. If the final definition is on a coarser level, the fraction of vessels with or without these devices can be specified, including the uncertainties.
The generic vessel system would look as depicted in Figure 3. It should be noted that all the barriers indicated have the purpose of preventing a loss of containment. Temperature and pressure could also be important for the quality of a product. The functions to maintain quality are not modelled.

Not all components need to be present. Because we use continuous BBNs it is simple to "switch off" a node.

Currently no distinction is made between vessels and pipes with different materials. This can be done because the top event is probability of LOC (-size) and in this development the consequences need not be calculated. 


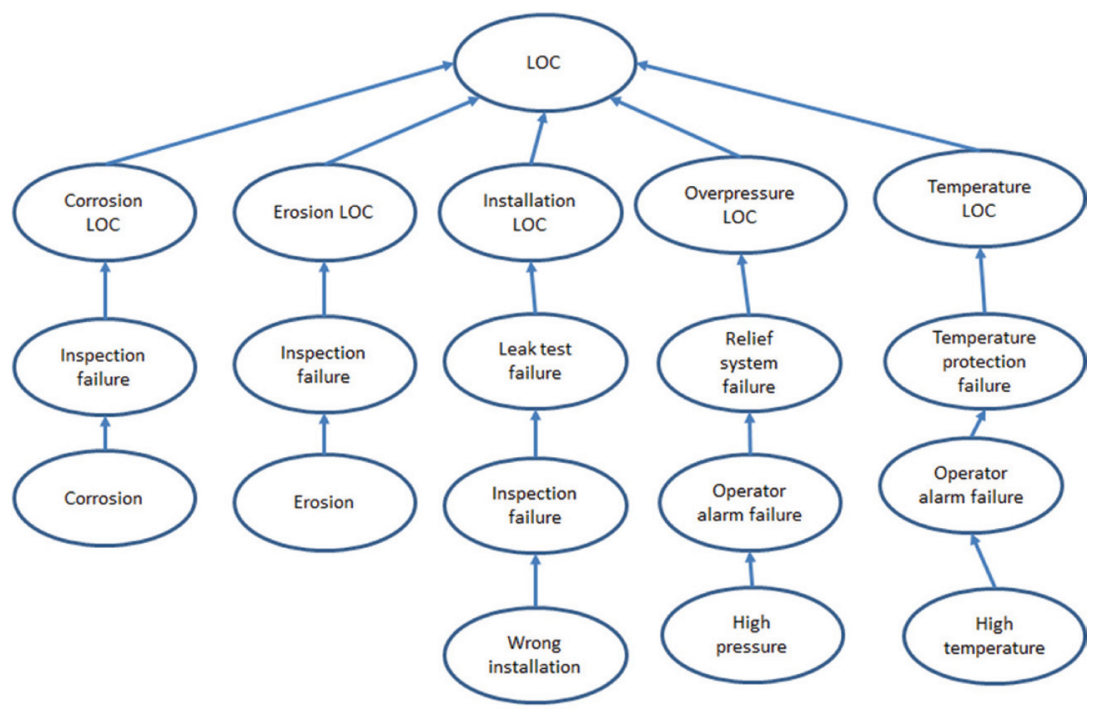

Figure 3. Generic BBN for a vessel or vessel-like equipment.

If this becomes necessary the distributions have to be restricted to vessels and pipes with the same or similar content, such as toxic, K1, K2, K3 hydrocarbons.

Similarly if the contents of a vessel can interact with the vessel wall special attention has to be given to these vessels, because generic modelling may not be justified. These vessels then need to be modelled in isolation. A similar argument holds for vessels in which catalytic and other chemical reactions take place and where mechanisms such as runaway can contribute significantly to the failure rate such that assuming them as members of the generic population is not justified.

A more detailed description of how the generic BBN's are combined in a specific case and how the $\mathrm{BBN}$ subsequently is populated with data and distributions is given in another paper in this conference (Gulijk et al. 2013).

\subsection{Pumps and valves}

Pumps and valves can fail in two ways: they could leak themselves and thus create a LOC but their failure to operate properly could also lead to over pressurization or overfilling of a vessel.

Therefore these pumps and compressors are modelled as vessels for their LOC and in the BBN certain parameter excursions, which are not fatal for the piece of equipment itself is connected to an initiation event for other vessel when required.

Valves are for their LOC treated as pieces of pipework. Their functional failure is part of de Safeguards for which they serve.

\section{PIPE STRUCTURE}

For pipes the generic failure causes are overpressure, overheating, corrosion and impact. It is unusual to have barriers against temperature and pressure excursions on the pipes. They are usually located at the vessels at the end. So what remain is corrosion and impact. Again this is achieved by using the same basic Vessel BBN and switching off the appropriate nodes.

\section{SPECIFICATION OF THE UNIT}

A unit consists of vessels, pipes and miscellaneous equipment not already included in the vessel structure.

In the most detailed specification each vessel is specified separately by position content and type. It is also possible to define these as distributions.

It is advisable not to describe all vessels of all types as a single distribution, because their base failure rate according to the Purple Book is different. For each type of vessel a distribution over size and over the other parameters can be given. If these distributions are correlated, the correlations can be given.

The pipes can be specified as a distribution of their length over their diameter. Pumps, flanges, welds, bends and bellows, which are "along the pipe" and cannot be modelled in the vessel structure can be modelled with the pipe or as an average addition to the pipe length. Note that according to the Purple Book the base failure rate is correlated 
with the diameter. For this correlation a continuous function can be used rather than the 3 point distribution also specified in the Purple Book.

\section{SPECIFICATION OF A PLANT AND A SITE}

The plant as a whole can be defined/modelled by specifying each unit separately and add the inter unit pipes to the specification. Alternatively the plant can be modelled as a population of units. The units would then be defined by the probability of having a certain number of vessels, compressors, length of pipe etc. In that latter case the granularity on unit level is lost.

Similarly a whole site can be modelled as site wide distributions of plants, although on such a course level no meaningful results can be expected.

\section{HUMAN FACTORS}

In Ale et al (2009 a, b) we described how the structure of the human behaviour model can be used to represent the various human actors involved in the operation. In the current implementation of the method we distinguish two types of people: the companies own personnel and the personnel hired through contractors (Lin et al. 2013). This distinction has been made because personnel hired by contractors are under the managerial influence not only of Shell but also of the contractor company by which they are employed. The structure of the human model and its development is described in more detail by Lin et al (2012).

For this project the motivational issues driving the behaviour of the people involved and the differences between the two groups have been investigated in more detail, among other by extensive inquiries among personnel of one of the sites of Shell. (Ale et al 2012b, Sillem et al. 2013).

The generic BBN for human behaviour is inserted whenever a human action is involved. This can be by direct action e.g. where an operator serves as the logic processor and actuator of a barrier. It could also be by indirect action such as wrong specification of materials or other design errors.

The influence of management is modelled through connections to the human performance model as was done before in the CATS project and in the WORM project (Ale 2006).

\section{COMPUTER PROGRAM}

For a specific plant of Shell the model has been implemented. In order to do so special software has been developed based on the Uninet software of TU-Delft. This program handles the conversion of the company's inventory lists into the appropriate generic BBN structures. It guides the user through the specification of items, it connects the models for equipment to the models for humans and connects the management layer where appropriate. Because we use repetitive generic structures the first cut on building the BBN for a plant can be automated. While doing so the program builds and keeps a database of all the numbers used in the program including their pedigree. This book- and record keeping continues when the analyst starts to detail the structure with dedicated specifications of items and when managerial interventions are studied for their potential effect. Because we also developed a method to model the behaviour of the BBN over time (Hanea 2012) it is now possible to estimate when the results of certain management actions, such as safety promotion campaigns can be expected to become visible. This in turn gives information on for instance how long certain campaigns need to be supported to have a chance of success and thus how much money will be involved before success becomes visible.

\section{CAVEATS}

The whole of the project is aimed at identifying the potential for disaster, which lies in the extremes of the distributions of probability and effect. Up to this project risk analyses were based on average base frequencies derived from historical data. The problem long since recognized is that this gives only a rough indication and is insufficient for improving safety beyond the current level. The average chemical plant does not blow up and the average oil rig does not sink. It is the infrequent, but nevertheless possible combination of extremes that gives rise to a catastrophe.

The analysis tool supports decisions whether or not it is wise to spend money on reducing these probabilities or take them away.

It does not take away the problem that any analysis depends on the quality of the information, in this case the width of the distributions entered for the parameters. In other more simple terms: whether the analyst beliefs that certain excursions are possible.

Although this is often referred to as the "Black Swan" problem, this problem is much harder than that. It is more like the "Fire Breathing Dragon" problem. There are libraries full of books about these dragons, so it cannot be ignored that the possibility of their existence is raised. It is abundantly clear from this literature that having a dragon breathing fire in a chemical plant is not a good idea. On the other hand, as far as we know, 
nobody has seen one yet. The first question now is do we have to consider the construction of an Anti Dragon Defence Shield (ADDS). The second question is what we are going to say if we do not construct the ADDS and a dragon appears. This problem cannot be solved by any form of technical analysis, be it frequentistic or Bayesian.

On the other hand, there are not many occasions where an accident or disaster is really completely new. May be the specific combination of factors has not occurred previously, but all the elements and the extent of possible excursions were known. What lacked was a method to harness this knowledge in a usable way. The current development tries to contribute to a further exploitation of collective knowledge.

\section{CONCLUSION}

A computer assisted method to perform a quantified risk analysis, that includes the variability of probabilities, the development of failure mechanisms over time, the performance of human beings and the influence of management in such a way that it can effectively, in a timely manner and without excessive analytical effort support management decisions on investments in risk reducing measures was successfully developed and applied in an existing chemical plant.

\section{ACKNOWLEDGEMENT}

This work reported above was fully funded by Royal Dutch Shell plc.

\section{REFERENCES}

Ale B.J.M. \& Whitehouse, R., 1984. A computer based system for risk analysis of process plants. In Heavy Gas and Risk Assessment III, 5. Hartwig (Ed) D. Reidel, Dordrecht, The Netherlands. November 1984.

Ale, B.J.M. \& Uijtdehaag, P.A.M. 1999. Guidelines for Quantitative Risk Analysis, (CPR18) RIVM, SDU, Den Haag, ISBN 90-12-08796-1.

Ale, B.J.M. 2006. The Occupational Risk Model, TUDelft/TBM RC 20060731, ISBN 90-5638-157-1, Delft, 2006.

Ale, B.J.M., Bellamy, L.J., Boom, R van der, Cooper, J., Cooke, R.M., Goossens, L.H.J., Hale, A.R., Kurowicka, D., Morales, O., Roelen, A.L.C., Spouge, J. 2009a, Further Development of a Causal model for Air Transport Safety (CATS); building the mathematical heart, Reliability Engineering and System Safety 94 (2009) 1433-1441.
Ale, B., Bellamy, L.J., Cooke, R.M., Duyvis, M., Kurowicka, D., Lin, P.H., Morales, O., Roelen, A.L.C., Spouge, J.. 2009b. Causal Model for Air Transport Safety-Final report. Ministerie van Verkeer en Waterstraat, Directoraat-Generaal Luchtvaart en Maritieme zaken. ISBN 13: 97890369 1724-7.

Ale, B.J.M., Hanea, D., Gulijk, C. van, Lin, P.-H., Sillem, P.-H., Hudson, P., 2011. Towards an integrated risk model for hydrocarbon industry operation, Proceedings of the European Safety and Reliability Conference, ESREL 2011 18-22 September 2011Troyes France, Advances in Safety, Reliability and Risk Management-Bérenguer, Grall \& Guedes Soares (eds), Taylor \& Francis Group, London, ISBN 9780-415-68379-1.

Ale, B.J.M., Hanea, D, Sillem, S, Lin, P-H, Gulijk, C. van, Hudson, P. 2012a. Modelling risk in high hazard operations: integrating technical, organisational and cultural factors; PSAM 11, Esrel 2012, Helsinki 25-29 juni 2012.

Ale, B.J.M. Sillem, S., Lin, P-H., Hudson, P. 2012b. Modelling human and organizational behaviour in a high-risk operation; PSAM 11, Esrel 2012, Helsinki 25-29 juni 2012.

Beddington, J., 2011. Blacket Review of Hight Impact Low Probability Risks, Crown Copyright 2011, UK, URN $12 / 519$.

Christou, M., 2008. Major Accidents: Examples, statistics and remarks on their prevention, XI EWHN Conference, 11 October 2008, www.ewhn.eu/attachments/article/.../Workshop3CRISPRE SENTATION. pdf (23-03-2011).

Gulijk, C van., Hanea, D., Steenhoek, B., Marcel, Karina Q. Almeida-Leñero, K.Q., Ale, B.J.M. 2013. developing hazard control path from industrial data..ESREL 2013 (This conference)

Hanea, D., Hanea, A., Ale, B.J.M., Sillem, S, Lin, P-H., Gulijk C. van, Hudson, Patrick. 2012. Using Dynamic Bayesian Networks to Implement Feedback in a Management Risk Model for the Oil Industry; PSAM 11, Esrel 2012, Helsinki 25-29 juni 2012.

Hudson, P.TW., (2010. Safety Science: It's not rocket science, it's much harder. Inaugural address T.U. Delft. 24 September 2010.

Lin, P-H., Hanea, D., Ale, B.J.M., Sillem, S., Gulijk, C., van, Hudson, P. 2012. Integrating organisational factors into a BBN model of risk; PSAM 11, Esrel 2012, Helsinki 25-29 juni 2012

Lin, P-H, Hanea, D, Ale, B.J.M. 2013. Modeling contractor and company employee behavior in high hazard operation, esrel 2013 (this conference).

Sillem, S., Lin, P-H, Ale, B.J.M. 2013. Modelling human motivation in a high-risk operation. ESREL 2013 (This conference).

Uijt de Haag, P.A.M., Ale, B.J.M.. Post, J.G. 2000. Guideline for quantitative risk assessment: Instructions for a quantitative risk analysis in The Netherlands in G.I. Schuller and P. Kafka (eds) Safety and Reliability. ISBN 905809109 0, Balkema, Rotterdam, The Netherlands. 